\title{
PROJETO TERAPÊUTICO SINGULAR: FERRAMENTA DE SUPERAÇÃO DO GAP TERAPÊUTICO EM SAÚDE MENTAL
}

Francisco João de Carvalho Neto ${ }^{1}$ Maria da Clória Sobreiro Ramos ${ }^{1}$ Mayla Rosa Guimarães ${ }^{1}$

Aline Raquel de Sousa Ibiapina ${ }^{1}$ Ana Paula Cardoso Costa ${ }^{1}$ Rávida da Rocha Lima Silva ${ }^{2}$ Lany Leide de Castro Rocha Campelo ${ }^{1}$ Girzia Sammya Tajra Rocha ${ }^{1}$

\author{
https://orcid.org/0000-0002-2011-5900 \\ https://orcid.org/0000-0001-7847-7077 \\ https://orcid.org/0000-0002-6501-7853 \\ https://orcid.org/0000-0003-1373-3564 \\ https://orcid.org/0000-0002-1550-3685 \\ https://orcid.org/0000-0001-9738-7077 \\ https://orcid.org/0000-0002-1686-9312 \\ https://orcid.org/0000-0002-1624-3838
}

Objetivo: Relatar a experiência acadêmico-assistencial de estudantes de Enfermagem durante a construção conjunta de um projeto terapêutico singular com as equipes de atenção à saúde da familia em uma Unidade Básica de Saúde. Metodologia: Estudo descritivo do tipo relato de experiência sobre a elaboração de um projeto terapêutico singular, a partir das experiências vividas por acadêmicos de Enfermagem da Universidade Federal do Piauí no segundo semestre de 2019 ao acompanhar uma paciente com transtorno mental. Resultados: Como resultado do seguimento das etapas diagnóstico situacional, definição de metas, divisão de responsabilidades e avaliação e reavaliação, bem como do engajamento dos acadêmicos e profissionais e da paciente e sua família em cada fase do projeto terapêutico singular, foi possivel identificar os aspectos orgânicos, psicológicos e sociais desencadeadores dos problemas e riscos à saúde e planejar e implementar intervenções voltadas ao suprimento das demandas da paciente e familia, consequentemente melhorando sua qualidade de vida. Conclusões: O projeto terapêutico singular demonstrou ser uma ferramenta importante para a superação do distanciamento terapêutico existente entre paciente, familia e equipe, contribuindo para o fortalecimento dos vínculos, uma melhor resolutividade das demandas do paciente e família e a produção de sua autonomia quanto ao seu autocuidado.

Descritores: Atenção Primária à Saúde; Planejamento em Saúde; Saúde Mental.

\section{SINGULAR THERAPEUTIC PROJECT: TOOL FOR OVERCOMING THERAPEUTIC GAP IN MENTAL HEALTH}

Objective: To report the experience of academic care of nursing students during the joint construction of a singular therapeutic project with the family health care teams in a Basic Health Unit. Methodology: A descriptive study of the type of experience report on the elaboration of a singular therapeutic project. based on the experiences lived by Nursing students of the Federal University of Piaui in the second semester of 2019 when they followed up on a patient with mental disorder was carried out. Results: As a result of following the stages of situational diagnosis, goal setting, division of responsibilities, and evaluation and reevaluation, as well as the link between academics and professionals with the patient and her family in each phase of the singular therapeutic project. it was possible to identify the organic, psychological and social aspects that trigger health problems and risks to plan and implement interventions aimed at satisfying the demands of the patient and their family, consequently improving their quality of life. Conclusions: The singular therapeutic project proved to be an important tool to overcome the existing therapeutic gap between patient, family and health team, contributing to the strengthening of ties, a better resolution of the demands of the patient, the family and the production of autonomy and personal care.

Descriptors: Primary Health Care; Health Planning; Mental Health.

\section{PROYECTO TERAPEUTICO SINGULAR: HERRAMIENTA PARA SUPERAR EL DISTANCIAMIENTO TERAPÉUTICO EN LA SALUD MENTAL}

Objetivo: Relatar la experiencia de atención académica de estudiantes de Enfermería durante la construcción conjunta de un proyecto terapéutico singular con los equipos de atención a la salud familiar en una Unidad Básica de Salud. Metodología: Estudio descriptivo del tipo relato de experiencia sobre la elaboración de un proyecto terapéutico singular, a partir de las experiencias vividas por estudiantes de Enfermeria de la Universidad Federal de Piaui en el segundo semestre de 2019 cuando realizaban seguimiento a una paciente con trastorno mental. Resultados: Como resultado de seguir las etapas de diagnóstico situacional, establecimiento de objetivos, división de responsabilidades y evaluación y reevaluación, así como la vinculación de académicos y profesionales con la paciente y su familia en cada fase del proyecto terapéutico singular, fue posible identificar los aspectos orgánicos, psicológicos y sociales que desencadenan problemas y riesgos a la salud para planificar e implementar intervenciones dirigidas a satisfacer las demandas del paciente y su familia, mejorando como consecuencia su calidad de vida. Conclusiones: El proyecto terapéutico singular demostró ser una herramienta importante para superar el distanciamiento terapéutico existente entre paciente, familia y equipo de salud, contribuyendo al fortalecimiento de los vínculos, una mejor resolución de las demandas del paciente, la familia y la producción de su autonomía con respecto a sus cuidados personales.

Descriptores: Atención Primaria de Salud; Planificación en Salud; Salud Mental.

'Universidade Federal do Piaui, PI, Brasil.

2Universidade Federal do Ceará, CE, Brasil.

Autor correspondente: Ana Paula Cardoso Costa - Email: anapaulaccardoso@hotmail.com

Recebido: 28/03/2020 - Aceiro: 18/08/2020 


\section{INTRODUÇÃO}

A Reforma Psiquiátrica é um processo político e social complexo, composto por atores, instituições e forças de diferentes origens, e que reflete em todos os âmbitos da sociedade. Compreendida como um conjunto de transformações de práticas, saberes, valores culturais e sociais, foi estabelecida com o intuito de humanizar as condições de tratamento e cuidado às pessoas que sofrem com transtornos mentais, rompendo com o modelo hegemônico até então vigente'.

Resultado desse movimento foi a promulgação da Lei no 10.2016/2001, na qual foi oficializado o atendimento psiquiátrico humanizado, a proteção às pessoas com transtornos psiquiátricos, a preferência pelos serviços comunitários sobre a internação, a implantação em todo território nacional de serviços substitutivos, as bases de funcionamento desses serviços e a regulamentação das internações compulsórias².

Apesar dos avanços relacionados à assistência, as práticas de cuidado em saúde mental no Brasil seguem pautadas em ações realizadas no próprio serviço, sem articulação com o território e com enfoque no tratamento medicamentoso dos sintomas do transtorno mental. Observa-se ainda, a ausência de um olhar para as especificidades e singularidades da pessoa que sofre e a reprodução de um cuidado intramuros característico do modelo asilar, divergindo do que propõe a Reforma Psiquiátrica ${ }^{3}$.

Um dos modelos de assistência para romper com a conjuntura asilar são os Centros de Atenção Psicossocial (CAPS), que se constituem pontos de atenção estratégicos da Rede de Atenção Psicossocial (RAPS), sendo serviços de saúde de caráter aberto e comunitário, constituídos por equipe multiprofissional que atua sobre a ótica interdisciplinar no atendimento às pessoas com sofrimento ou transtorno mental, incluindo aquelas com necessidades decorrentes do uso de álcool e outras drogas ${ }^{4.5}$.

A Atenção Primária à Saúde (APS) é porta de entrada para 0 atendimento de pessoas com transtornos mentais no sistema de saúde. Sua finalidade é promover ações de saúde para o indivíduo e coletividade, abrangendo a promoção e proteção da saúde, prevenção de agravos, diagnóstico, tratamento, reabilitação, redução de danos e manutenção da saúde, oferecendo uma atenção integral a seus usuários ${ }^{6}$.

Nesse cenário, os Núcleos de Apoio à Saúde da Família (NASF) se constituem como serviços que ampliam a abrangência e o escopo das ações da Atenção Básica (AB), bem como sua resolutividade. Devem atuar de forma integrada à Rede de Atenção à Saúde e seus serviços, responsabilizando-se e compartilhando suas ações com as equipes da Estratégia de Saúde da Família (ESF) buscando contribuir para a integralidade do cuidado prestado?.

Algumas estratégias têm potencial de reduzir substancial- mente o distanciamento terapêutico de pessoas em sofrimento psíquico e a APS e, consequentemente, a carga de doença produzida pelos transtornos mentais: a integração da atenção à saúde mental aos serviços de APS; o compartilhamento e delegação de tarefas; e a incorporação de inovações tecnológicas aos modelos de ofertas de serviços existentes de saúde mental ${ }^{8}$.

Uma ferramenta para efetivar a superação desse gap terapêutico em saúde mental, ou seja, a lacuna existente entre pessoas com transtornos mentais e APS, é o Projeto Terapêutico Singular (PTS), definido como um conjunto de propostas de condutas terapêuticas articuladas, para um sujeito individual ou coletivo, resultado da discussão coletiva de uma equipe interdisciplinar, com apoio matricial ${ }^{9}$. Nesse contexto, o PTS se configura como um movimento de coprodução e cogestão do processo terapêutico que promove integração entre as equipes de saúde ${ }^{10}$.

Frente ao exposto, o presente estudo tem por objetivo relatar a experiência acadêmico-assistencial de estudantes de Enfermagem durante a construção conjunta de um PTS com as equipes ESF e NASF em uma Unidade Básica de Saúde (UBS).

\section{METODOLOGIA}

\section{Tipo de estudo}

Trata-se de um estudo descritivo, do tipo relato de experiência, sobre a elaboracão de um PTS por acadêmicos de Enfermagem da Universidade Federal do Piauí (UFPI).

\section{Cenário do estudo}

O estudo foi desenvolvido em uma UBS situada no município de Picos, Piauí, Brasil, durante visitas domiciliares a uma paciente assistida por uma das ESF da unidade entre os meses de agosto e outubro de 2019.

\section{Sujeitos envolvidos na experiência}

A experiência contou com participação de acadêmicos de enfermagem e docentes preceptores da UFPI, profissionais da ESF, NASF e CAPS, e paciente para o qual foi elaborado e implementado o PTS relatado.

\section{Aspectos éticos}

Por tratar-se de um relato de experiência dos autores em atividade acadêmica realizada em um estágio curricular, não houve necessidade de submissão à apreciação do Comitê de Ética em Pesquisa. Ressalta-se, no entanto, que se mantiveram os principios éticos durante toda a execução do plano terapêutico e descrição da atividade neste artigo.

\section{Objetivos da Experiência}

A experiência teve como objetivos construir e imple- 
mentar um PTS para uma paciente com transtorno mental assistida na APS.

\section{Descrição da experiência}

Durante o periodo do estágio foi possivel conhecer a rotina do serviço e as demandas da população atendida. A partir de então pôde-se identificar uma situação complexa envolvendo uma paciente portadora de transtorno mental sem acompanhamento especializado e em situação de extrema vulnerabilidade.

Em cumprimento das etapas diagnóstico situacional, definição de metas, divisão de responsabilidades, avaliação e reavaliação, a elaboração do PTS teve início com o diagnóstico situacional da paciente e família.

Devido a situação de saúde e a vulnerabilidade socioeconômica da paciente, foram realizadas visitas domiciliares juntamente com os profissionais do NASF, nas quais foram coletados os dados necessários à elaboração do plano terapêutico?.

Procurou-se conhecer a usuária, sua família, bem como o contexto social em que estavam inseridos para que se pudesse estabelecer uma relação de vínculo entre estes e a UBS, ferramenta importante na adesão ao plano terapêutico, bem como para o êxito no seu seguimento, tendo em vista que o vínculo se faz imprescindivel na construção de relações de afetividade e confiança entre usuário e profissional, alicerce para o aprofundamento no processo de corresponsabilização6.

Ainda na ocasião da primeira visita, os acadêmicos apresentaram à família a proposta de realização do PTS, e somente após o aceite deu-se início à intervenção com a continuidade das visitas e etapas subsequentes. É valido ressaltar que durante a execução do PTS todos apresentaram-se receptivos e colaborativos e que a inclusão da família foi fundamental, uma vez que a usuária era dependente da irmã para deslocar-se quando necessário.

Para desempenhar as metas propostas foram realizadas um total de cinco visitas domiciliares com a equipe multiprofissional, cujos objetivos foram esclarecidos. Nestas visitas, executaram-se atividades variadas conforme as necessidades da paciente/família, sempre buscando o fortalecimento dos vínculos ${ }^{11}$, principalmente devido a dificuldade de locomoção da paciente, e seu quadro de humor deprimido, fatores que poderiam dificultar a sua ida ao serviço de saúde, devido à falta de estímulo para busca de assistência ${ }^{12}$.

Diante disso, foi possível realizar o diagnóstico situacional e, assim, elencar os principais problemas existentes: vínculo deficiente com a equipe de saúde da UBS, tratamento psiquiátrico inadequado, falta de vínculo com o CAPS, isolamento social, nutrição inadequada, falta de cuidados com a saúde bucal. Foram identificados, também, riscos para desenvolvimento de outros problemas de saúde, tais como depressão e comporta- mento suicida. Observou-se ainda, déficit no autocuidado devido à falta de conhecimento e à condição de saúde mental e renda isuficiente, sugerindo outros riscos à saúde.

Após essa etapa, buscou-se na literatura informações referentes à doença, tratamentos, formas de reintegração social e articulação entre os serviços de apoio dentro da Rede de Atenção à Saúde do próprio município. Com as informações necessárias e equipe multidisciplinar disponível, foram elaboradas metas, responsabilidades e prazos a serem alcançados junto à paciente e família, assim como demonstrado no Quadro 1.

\section{Principais resultados alcançados}

Quadro 1 - Plano terapêutico elaborado por acadêmicos de Enfermagem, docentes preceptores, equipe ESF e NASF. Picos - PI, Brasil. 2019.

\begin{tabular}{|c|c|c|c|}
\hline Diagnóstico & Metas & $\begin{array}{l}\text { Responsá- } \\
\text { veis }\end{array}$ & Tempo \\
\hline \multirow{8}{*}{$\begin{array}{l}\text { Paciente de } 39 \text { anos, } \\
\text { sexo feminino, parda, } \\
\text { ensino fundamental } \\
\text { incompleto, reside } \\
\text { com sua irmã e dois } \\
\text { sobrinhos adolescen- } \\
\text { tes. Renda familiar } \\
\text { oriunda de beneficio } \\
\text { da paciente (Loas). Há } \\
\text { alguns anos, princi- } \\
\text { palmente devido ao } \\
\text { seu comportamento } \\
\text { agressivo, e compulsão } \\
\text { sexual, a familia buscou } \\
\text { tratamento no CAPS, } \\
\text { onde foi diagnosticado } \\
\text { também um retardo } \\
\text { mental moderado (CID } \\
\text { F7l.l). Devido à falta } \\
\text { de informação, baixo } \\
\text { nível socioeconômico } \\
\text { da familia, e dificuldade } \\
\text { de acesso ao serviço, a } \\
\text { paciente não deu con- } \\
\text { tinuidade ao acompa- } \\
\text { nhamento psiquiátrico, } \\
\text { mantendo a prescrição } \\
\text { inicial ("Diazepan") sem } \\
\text { nenhuma reavaliação, } \\
\text { mesmo sem a melhora } \\
\text { do quadro. } \\
\text { Ao exame fisico, } \\
\text { constatou-se dentição } \\
\text { prejudicada relacionada } \\
\text { à barreira ao autocui- } \\
\text { dado e conhecimento } \\
\text { insuficiente. } \\
\text { Isolamento social, } \\
\text { interação social preju- } \\
\text { dicada, risco de solidão. } \\
\text { Em sua residência, a } \\
\text { paciente é mantida } \\
\text { pela familia em cárcere } \\
\text { privado, em quarto } \\
\text { escuro, com grades e } \\
\text { sem ventilação. }\end{array}$} & $\begin{array}{l}\text { Visita domiciliar para } \\
\text { consulta de acolhimen- } \\
\text { to e cadastramento, } \\
\text { objetivando melhorar o } \\
\text { vínculo com a UBS. }\end{array}$ & $\begin{array}{l}\text { Enfermeira, } \\
\text { acadê- } \\
\text { micos e } \\
\text { docentes } \\
\text { precepto- } \\
\text { res. }\end{array}$ & $\begin{array}{l}\text { Ime- } \\
\text { diato }\end{array}$ \\
\hline & $\begin{array}{l}\text { Encaminhamento ao } \\
\text { CAPS, para retomada } \\
\text { do acompanhamento } \\
\text { psiquiátrico. }\end{array}$ & $\begin{array}{l}\text { Enfermeira } \\
\text { Psiquiatra, } \\
\text { psicólogo } \\
\text { e equipe } \\
\text { multipro- } \\
\text { fissional }\end{array}$ & $\begin{array}{l}\text { Ime- } \\
\text { diato }\end{array}$ \\
\hline & $\begin{array}{l}\text { Encaminhamento à } \\
\text { assistência social, para } \\
\text { avalição da situa- } \\
\text { ção socioeconômica } \\
\text { familiar. }\end{array}$ & $\begin{array}{l}\text { Assistente } \\
\text { social }\end{array}$ & $\begin{array}{l}\text { Pri- } \\
\text { meiro } \\
\text { mês }\end{array}$ \\
\hline & $\begin{array}{l}\text { Elaboração de um } \\
\text { plano alimentar e } \\
\text { acompanhamento } \\
\text { nutricional. }\end{array}$ & $\begin{array}{l}\text { Nutricio- } \\
\text { nista }\end{array}$ & $\begin{array}{l}\text { Pri- } \\
\text { meiro } \\
\text { mês }\end{array}$ \\
\hline & $\begin{array}{l}\text { Assistência médica } \\
\text { para avaliação geral do } \\
\text { estado de saúde. }\end{array}$ & Médico & $\begin{array}{l}\text { Pri- } \\
\text { meira } \\
\text { sema- } \\
\text { na / } \\
\text { conti- } \\
\text { nuo }\end{array}$ \\
\hline & $\begin{array}{l}\text { Acompanhamento com } \\
\text { fisioterapeuta, visto } \\
\text { que paciente se apre- } \\
\text { sentava em situação de } \\
\text { mobilidade dificultada } \\
\text { devido isolamento em } \\
\text { um quarto. }\end{array}$ & $\begin{array}{l}\text { Fisiotera- } \\
\text { peuta }\end{array}$ & $\begin{array}{l}\text { Men- } \\
\text { sal }\end{array}$ \\
\hline & $\begin{array}{l}\text { Visitas domiciliares } \\
\text { mais frequentes para } \\
\text { o desenvolvimento de } \\
\text { vínculo paciente/equi- } \\
\text { pe e acompanhamento } \\
\text { da situação de saúde. }\end{array}$ & $\begin{array}{l}\text { Agente co- } \\
\text { munitário } \\
\text { de saúde, } \\
\text { enfermeiro, } \\
\text { médico e } \\
\text { assistente } \\
\text { social. }\end{array}$ & $\begin{array}{l}\text { Conti- } \\
\text { nuo }\end{array}$ \\
\hline & $\begin{array}{l}\text { Avaliação da saúde } \\
\text { bucal. }\end{array}$ & Dentista & $\begin{array}{l}\text { Men- } \\
\text { sal }\end{array}$ \\
\hline
\end{tabular}

Fonte: Autores. 
A etapa subsequente do projeto se constituiu na avaliação de alcance das metas estabelecidas conjuntamente entre equipes ESF e NASF, conforme demonstrada no Quadro 2.

Quadro 2 - Avaliação das metas estabelecidas no PTS por acadêmicos de Enfermagem, docentes preceptores, equipe eSF e NASF. Picos - PI, 2019.

\begin{tabular}{|c|c|}
\hline $\begin{array}{l}\text { Profissionais } \\
\text { Responsáveis }\end{array}$ & Metas Alcançadas \\
\hline Enfermeira & $\begin{array}{l}\text { Melhor vínculo com a equipe da UBS, NASF e CAPS. } \\
\text { Paciente encaminhado ao CAPS II para realização } \\
\text { de consulta com psiquiatra, realizado diagnóstico e } \\
\text { sugerido tratamento na modalidade não intensiva, } \\
\text { de frequência mensal ou conforme necessidade. A } \\
\text { partir da consulta com o psiquiatra, passou a fazer } \\
\text { uso das seguintes medicações: Neozine® } 25 \text { mg. } \\
\text { Zargus® } 2 \text { mg e Fenergan® } 25 \mathrm{mg}\end{array}$ \\
\hline Assistente social & Realizado cadastro único \\
\hline Nutricionista & $\begin{array}{l}\text { Plano alimentar elaborado conforme condição } \\
\text { econômica }\end{array}$ \\
\hline Psicóloga & Acompanhamento quanto às demandas psiquicas \\
\hline Médica & $\begin{array}{l}\text { Prestada assistência médica durante o tratamento } \\
\text { e acompanhamento para reavaliação e renovação } \\
\text { de receitas }\end{array}$ \\
\hline ACS & Maior frequência de visitas ao domicilio \\
\hline Fisioterapeuta & $\begin{array}{l}\text { Acompanhamento com fisioterapeuta devido situa- } \\
\text { ção de mobilidade prejudicada }\end{array}$ \\
\hline Dentista & $\begin{array}{l}\text { Avaliação da saúde bucal realizada e tratadas as } \\
\text { demandas }\end{array}$ \\
\hline
\end{tabular}

Evidenciou-se que a integração entre ESF, NASF e acadêmicos de enfermagem foi imprescindivel para que as metas propostas fossem alcançadas com êxito. Além disso, a criação de vínculos permitiu aproximação entre os envolvidos no plano terapêutico, otimizando o ato de cuidar ${ }^{13}$.

Ademais, notou-se que a incorporação do cuidado em saúde mental na APS pode exercer papel fudamental na diminuição do estigmal4. O matriciamento, compartilhamento e delegação de tarefas podem reduzir os custos e a quantidade de profissionais de saúde necessários para suprir as demandas no nível da APS. Inovações tecnológicas têm capacidade para expandir o alcance dos serviços, cortar custos e, também, reduzir o estigma. Dessa forma, estratégias como o PTS mostram-se eficazes e precisam ser exploradas pelos mais diversos profissionais da saúde ${ }^{15}$.

\section{Limitações da experiência}

Como limitação da experiência, aponta-se àquela relacionada ao não acompanhamento subsequente do paciente pelos acadêmicos de Enfermagem e docentes preceptores, em virtude do encerramento do período de estágio, para, assim, observar a continuidade da implementação do PTS.

\section{Contribuições para a prática}

A elaboração e implementação do PTS possibilitou a transformação do estado de saúde da paciente, viabilizou um maior conhecimento sobre o sofrimento mental, proporcionando a quebra de paradigmas e estigmas presentes em diversos contextos, e contribuiu para a articulação do cuidado biopsicossocial.

Acredita-se que o relato desta experiência possa contribuir para a ampliação de ações voltadas para a promoção da saúde mental no contexto da $A B$ e para que o PTS seja implementado e executado nesse contexto, visando à superação da lacuna terapêutica existente entre APS e pacientes com transtornos mentais, bem como otimizando à integralidade do cuidado.

\section{CONSIDERAÇÕES FINAIS}

A elaboração do PTS demonstrou ser uma ferramenta com potencial para superação do distanciamento terapêutico entre paciente, família e equipe de saúde, contribuindo para uma melhor integração destes e sendo eficaz na resolutividade de problemas. A experiência foi exitosa não só para os acadêmicos, mas também para os profissionais atuantes na UBS, que puderam visualizar o contexto da usuária em sua integralidade e os benefícios advindos após execução do PTS.

Durante as visitas domiciliares, verificou-se o quanto é necessária a ligação entre profissional de saúde e usuário. Alguns individuos podem encontrar-se impossibilitados de frequentar o serviço de saúde, assim, a visita domiciliar supera essa dificuldade na mobilidade, e, para além disso, possibilita visualizar problemas encontrados no âmbito familiar.

\section{Contribuições dos autores:}

FJC: concepção e/ou desenho do estudo; coleta, análise e interpretação dos dados; redação e/ou revisão crítica do manuscrito; aprovação da versão final a ser publicada. MGSR: concepção e/ou desenho do estudo; coleta, análise e interpretação dos dados; redação e/ou revisão crítica do manuscrito; aprovação da versão final a ser publicada. MRG: concepção e/ou desenho do estudo; coleta, análise e interpretação dos dados; redação e/ou revisão crítica do manuscrito; aprovação da versão final a ser publicada. ARSI, APCC, RRLS, LLCRC, GSTR: redação e/ou revisão crítica do manuscrito; aprovação da versão final a ser publicada. 


\section{REFERÊNCIAS}

1 Ramos DKR, Paiva IKS, Guimarães J. Pesquisa qualitativa no contexto da Reforma Psiquiátrica brasileira: vozes, lugares, saberes/fazeres. Ciênc saúde coletiva [Internet]. 2019; 24(3):839-852. Available from: https://doi.org/10.1590/141381232018243.00512017

2 Souza SAN, Santos MWL, Santos EGM. A Reforma Psiquiátrica na concepção e prática de uma equipe transdisciplinar de profissionais da saúde em um centro de convivência. Revista JRG De Estudos Acadêmicos [Internet]. 2020; 3(6):28-35. Available from: http://www.revistajrg.com/index.php/jrg/article/view/107

3 Campos DB, Bezerra IC, Jorge MSB. Produção do cuidado em saúde mental: práticas territoriais na rede psicossocial. Trab educ saúde [Internet]. 2020; 18(1):1-18. Available from: https://doi.org/10.1590/1981-7746-sol00231

4 Ministério da Saúde (BR). Portaria no 3.088, de 23 de dezembro de 2011 [Institui a Rede de Atenção Psicossocial para pessoas com sofrimento ou transtorno mental e com necessidades decorrentes do uso de crack, álcool e outras drogas, no âmbito do Sistema Único de Saúde - SUS]. Brasilia-DF. Diário Oficial da República Federativa do Brasil. 2011. Available from: http://bvsms.saude.gov.br/bvs/saudelegis/gm/2013/ prt3088_23_12_2011_rep.html [Acesso em: 12 out 2019].

5 Ministério da Saúde (BR) [Internet]. Centro de Atenção Psicossocial (CAPS) [acesso em 06 fev 2020]. 2017. Available from: https://www.saude.gov.br/noticias/693-acoes-e-programas/41146-centro-de-atencao-psicossocial-caps [Acesso em: 30 out 2019].

6 Ministério da Saúde (BR). Portaria no 2.436, de 21 de setembro de 2017 [Aprova a Política Nacional de Atenção Básica, estabelecendo a revisão de diretrizes para a organização da Atenção Básica, no âmbito do Sistema Único de Saúde - SUS]. Brasilia-DF. Diário Oficial da República Federativa do Brasil. 2017. Available from: http://bvsms.saude.gov.br/bvs/saudelegis/gm/2017/ prt2436_22_09_2017.html [Acesso em: 15 out 2019].

7 Ministério da Saúde (BR). Cadernos de Atenção Básica Saúde Mental. Secretaria de Atenção à Saúde. Departamento de Atenção Básica. Brasilia: Ministério da Saúde, 2013. Available from: http://bvsms.saude.gov.br/bvs/publicacoes/cadernos_atencao_basica_34_saude_mental.pdf [Acesso em: 24 nov 2019].
8 Rebello TJl, Marques A, Gureje O, Pike KM. Innovative strategies for closing the mental health treatment gap globally. Curr Opin Psychiatry [Internet]. 2014; 27(4):308-14. Available from: https://doi.org/10.1097/YC0.0000000000000068

9 Ministério da Saúde (BR). Clínica Ampliada, Técnico de Referência e Projeto Terapêutico Singular. Núcleo Técnico da Política Nacional de Humanização. $2^{a}$ ed. Brasilia: Ministério da Saúde, 2008. Available from: http://bvsms.saude.gov.br/bvs/ publicacoes/clinica_ampliada_2ed.pdf [Acesso em: 25 nov 2019].

10 Ministério da Saúde (BR). Caderno Humaniza SUS - Volume 1 - Formação e Intervenção. Secretaria de Atenção à Saúde. Política Nacional de Humanização. Brasilia: Ministério da Saúde, 2010. Available from: http://bvsms.saude.gov.br/bvs/ publicacoes/cadernos_humanizaSUS.pdf [Acesso em: 24 nov 2019].

11 Kayama M, Setoya N, Doyle C. Expanding use of nurse home visiting for community psychiatric care in Japan. Psychiatr Q [Internet]. 2020; 24. Available from: https://doi.org/10.1007/ sll126-020-09721-w

12 Neves R, Dimenstein M, Paulon S, Nardi H, Bravo O, Galvão VABM, et al. A saúde mental no sistema único de saúde do Brasil: duas realidades em análise. Avances en Psicología Latinoamericana [Internet]. 2012; 30(2):356-368. Available from: http://www.scielo.org.co/pdf/apl/v30n2/v30n2all.pdf

13 Catanni AN, Siqueira DF, Carmo DRP, Terra MG, Pillon SC. Percepção de profissionais de Enfermagem no cuidado as pessoas internadas em Unidade de Atenção Psicossocial. Res Soc Dev [Internet]. 2020; 9(1):e82911676. Available from: http://dx.doi.org/10.33448/rsd-v9il.1676

14 Garcia GDV, Zanoti-Jeronymo DV, Zambenedetti G, Cervo MR, Cavalcante MDMA. Healthcare professionals perception of mental health in primary care. Rev Bras Enferm [Internet]. 2020: 73(1):e20180201. Available from: http://dx.doi. org/10.1590/0034-7167-2018-02011

15 Baptista JA, Camatta MW, Filippon PG, Schneider JF. Singular therapeutic project in mental health: an integrative review. Rev Bras Enferm [Internet] 2020; 73(2):e20180508. Available from: http://dx.doi. org/10.1590/0034-7167-2018-0508 